\title{
Discurso del Sr. Presidente del Congreso Argentino de Cirugía ${ }^{1}$ Congress of the Asociación Argentina de Cirugía Presidential Address
}

\author{
Juan E. Álvarez Rodríguez
}

Análisis de las controversias, a propósito del $88^{\circ}$ Congreso Argentino de Cirugía

Quiero agradecer al Sr. Presidente, Dr. Alejandro de la Torre, su presentación y comentarios, que seguramente están sesgados por la amistad que nos une. ¡Muchas gracias!

En un momento tan especial para cualquiera de nosotros, como es el de presidir el Congreso Argentino de Cirugía, corresponde honrar las instituciones y a personalidades que han sido fundamentales para nuestro desarrollo profesional y como personas. En primer lugar, la Facultad de Medicina de la Universidad de Buenos Aires, donde estudié mi carrera y de la cual tengo el honor de ser Profesor y miembro de su Cuerpo Directivo. Agradezco la presencia del Sr. Decano, Profesor Dr. Sergio Provenzano, como también la de varios Consejeros y Secretarios de esa casa. El Hospital Gral. de Agudos Dr. Cosme Argerich. ¡Qué puedo decir de este hospital, que ha sido mi casa durante más de cuarenta años y donde ingresé como Residente de $1^{\mathrm{er}}$ año de Cirugía en 1970, para retirarme a fines del año 2015 como Jefe del Servicio de Cirugía!

Mi primer Jefe y maestro fue el Dr. Andrés Santas, por aquel entonces también Decano de la Facultad de Medicina. El Dr. Santas fue un eximio cirujano y miembro de la Asociación Argentina de Cirugía, líder en educación médica y un ferviente promotor e impulsor de la Residencia, como el mejor sistema de formación de posgrado. Santas no estaba solo, pues se hallaba acompañado por prestigiosos cirujanos a quienes quiero recordar mencionando los que fueron líderes de aquel grupo, los Dres. Horacio Achával Ayerza, Vicente Gutiérrez Maxwell y Julio Diez, que nos aconsejaron y orientaron en nuestros primeros pasos en la cirugía. Al Dr. Santas lo sucedió Clemente Morel, también Profesor de Cirugía, del cual aprendimos el interés por la fisiopatología de las enfermedades quirúrgicas y la investigación. También Morel se hallaba acompañado por destacados cirujanos, como los Dres. Domingo Filippin, Luis Gutiérrez (hermano de Vicente), Juan J. Naveiro, Patricio Welsh, Jorge Alberthal y Alejandro Latzina, entre otros, quienes formaban el equipo de cirujanos con el cual llegó al hospital. Morel fue sucedido por el Dr. Juan José Fontana, médico del Hospital Argerich, a quien debo agradecer el haberme incorporado como staff en su Servicio. Un numeroso grupo de cirujanos trabajaba en el Hospital Argerich con Fontana, quienes lucharon día a día con nosotros, los residentes, para enseñarnos a operar. Sus nombres recorren mi memoria y a algunos pueden verlos en esta proyección, que los muestra en la casa de uno de ellos, el Dr. Antonio Rodríguez.

Mi último Jefe fue el Dr. Alejandro Salvador Oría, de quien quiero decir que -además- fue compañero de Residencia, Jefe de Residentes y amigo. El Dr. Oría ha sido una de las personalidades más brillantes de la cirugía que he conocido. Fue el líder indiscutible de nuestro grupo: él orientó el Servicio a la cirugía hepato-bilio-pancreática y logró que el Hospital Argerich fuera un centro de referencia en la cirugía del páncreas. Alejandro no solo fue brillante intelectualmente sino también generoso, al compartir con todos nosotros su producción científica. Su recuerdo permanecerá imborrable en mi memoria.

A mis compañeros del hospital también les quiero agradecer, como a las numerosas camadas de residentes con las cuales hemos trabajado tantas décadas. iSi es cierto, como dicen, que uno llega hasta donde lo empujan, pues bien, aquí tienen ustedes a los responsables de mi presencia hoy en esta circunstancia! Al Dr. Horacio D’Agostino, invitado especial a este Congreso, a quien debo agradecer la invitación para concurrir como fellow a su Servicio de Radiología Intervencionista en San Diego, en la oportunidad en que fuera designado como Relator Oficial del Congreso Argentino de Cirugía. ¡Muchas gracias, Horacio!

A mis predecesores en este cargo, los Dres. Ricardo Torres, Juan Pekolj y Alejandro de la Torre, a quienes debo el haber sido elegido como candidato a la Vicepresidencia del $87^{\circ}$ Congreso de Cirugía. A los miembros del Comité Congreso, con quienes hemos trabajado intensamente en el último año, para lograr un congreso que esté al nivel de nuestros asociados. Al personal administrativo de la Asociación, así como a nuestro Director, el Dr. Martín Mihura, de cuyo compromiso y colaboración hemos sido testigos tanto quienes me precedieron como yo. Puedo asegurarles que sin su colaboración sería imposible organizar un congreso de estas características. A las empresas que año tras año nos acompañan y ayudan en la financiación de este evento, así como a la colaboración recibida del Ministerio de Salud de la Nación, de la Provincia de Santiago del Estero y del Museo Evita. ¡Muchas gracias! Final- 
mente un recuerdo a mi familia, comenzando por mis padres: a ellos les debo más que la vida. En especial a mi madre, que nos acompañó hasta los 94 años, totalmente lúcida e inteligente, siempre con un consejo sabio y oportuno. Gracias a mis hermanos, Justo, Eva y Blanca, con quienes formamos una unión indivisible. A mis hijas Constanza, Pilar, María y Eugenia, que son el patrimonio más importante que tengo. Y por último a mi querida mujer, Ingrid, que me acompaña y aguanta, y que me ha dado un regalo inesperado a esta altura de los acontecimientos, que se llama Eugenia. A todos ellos muchísimas gracias.

Como ustedes sabrán, el lema que hemos seleccionado para el desarrollo de este Congreso es "Controversias en Cirugía" y quisiera hacer, por lo tanto, algunas reflexiones a propósito de la importancia de las controversias en el campo del conocimiento. En este sentido, podemos analizar las controversias desde tres puntos de vista: el semántico, el filosófico y el pedagógico.

\section{Desde el punto de vista semántico}

La Real Academia Española (RAE), en su vigesimotercera edición publicada en el año 2014 en colaboración con las 22 corporaciones que integran la Asociación de Academias de la Lengua Española (ASALE), y en conmemoración de su tricentenario, dice textualmente: Controversia: Discusión de opiniones contrapuestas entre dos o más personas.

Poco es lo que podemos agregar o decir de esto que es una definición y por lo tanto le da significado al término controversia en la lengua de habla hispana.

\section{Desde el punto de vista filosófico}

Es interesante la perspectiva de algunos filósofos sobre el tema, y me ha llamado la atención, particularmente, la de Marcelo Dascal. Este es un filósofo contemporáneo, nacido en Brasil y radicado actualmente en Israel, donde es Profesor de la Universidad de Tel Aviv. Tiene una obra muy importante que abarca la historia de la filosofía, filosofía de la ciencia, filosofía de la mente, pragmática y ciencias cognitivas. En el aspecto que nos interesa, Dascal ha desarrollado una teoría denominada "Teoría de las Controversias".

Desde su perspectiva, lo primero que realiza es un "diagnóstico de situación" con respecto a la filosofía actual, y en particular a la filosofía de la ciencia, rama muy reciente de la filosofía que cobró identidad a comienzos del siglo XX, que es la incapacidad para explicar el progreso del conocimiento científico. Dascal considera que tanto la corriente del positivismo lógico o neopositivismo, como sus sucesores, no han podido formular criterios categóricos que permitan diferenciar un enunciado científico del que no lo es. Dicho en otras palabras, no han podido caracterizar la "cientificidad" o explicar la "racionalidad de la ciencia". El conocimien- to científico no tiene un desarrollo lineal, como creían los positivistas, ni evoluciona en forma continua, uniforme y en una única dirección, como tampoco hay un método científico que sea aplicable a todas las situaciones. Vale decir, que la defensa de un método científico, que desarrollan los epistemólogos, positivistas y neopositivistas, basado en las ciencias físico-naturales, capaz de asegurarnos el único acceso al conocimiento verdadero, no existe. Otros filósofos contemporáneos han relativizado el valor indiscutible del pensamiento positivista. Es así como Thomas Kuhn, físico y filósofo estadounidense, en su obra La estructura de las revoluciones científicas, propuso una visión nueva de cómo se desarrolla la ciencia. Acuñó el término "paradigma" como un conjunto de realidades científicas aceptadas universalmente, que sirve como marco de referencia para el planteo de problemas y sus posibles soluciones. Dentro del paradigma se desarrolla o tiene lugar la denominada "ciencia normal", y así evoluciona hasta que aparecen algunas "anomalías" que no pueden explicarse en el contexto del paradigma vigente. Se genera entonces una "ciencia revolucionaria" en busca de un nuevo paradigma, que termina por reemplazar al anterior y que nada tiene que ver con este, ni puede analizarse con los principios aceptados en el paradigma previo. De un modo similar y aún más audazmente, Paul Feyerabend en su ensayo "Contra el Método" cuestiona la hipótesis neopositivista o positivista lógica, proponiendo el "anarquismo metodológico y epistemológico". Para este filósofo austríaco, la existencia de una metodología científica de aceptación universal es sencillamente un contrasentido.

En tal contexto, Dascal -con gran imaginación y creatividad- propone un camino que considera clave para dilucidar por qué o gracias a qué se expande el conocimiento: el estudio de las controversias.

Para Marcelo Dascal, la ciencia avanza por la existencia de conflictos epistémicos o del conocimiento, que se dirimen a través de las controversias. Es en ellas donde debería buscarse la explicación del progreso científico, y no tratar de encontrar un sello que diferencie lo que es científico de lo que no lo es. De esta forma elaboró inicialmente una hipótesis que devino luego en una teoría llamada "Teoría de las Controversias".

Según Dascal, las controversias solo tienen lugar entre personas, dos o más, o grupos de personas, que se enfrentan en un diálogo, sin el cual no hay polémica. Como consecuencia de estos ejercicios, que denomina "intercambios polémicos", los problemas se aclaran, surgen nuevos resultados y progresa el conocimiento.

La herramienta fundamental a través de la cual intentará demostrar su teoría es la "Pragmática", entendiendo como tal una rama de la lingüística que estudia todo lo que aportan los hablantes y su contexto para la significación del discurso. Este es tal vez uno de los aspectos más controvertidos de la teoría de Dascal, 
puesto que centra su análisis, como experto en pragmática, solo en las intenciones comunicativas de los que participan, que él se encarga de rastrear y reconstruir, revelando el conocimiento que cada uno de ellos tiene del tema que se debate.

Dascal diferencia tres tipos de intercambios polémicos o dialógicos, a saber: la discusión, la disputa y la controversia.

En la discusión, los que intervienen comparten un marco teórico y conceptual en determinada disciplina y la polémica se centra en temas bien delimitados. Durante su transcurso, una de las partes reconoce la existencia en su planteo de un error conceptual o metodológico y la discusión se resuelve.

En la disputa también se parte de posiciones contrapuestas, pero ninguno de los que intervienen acepta la posibilidad de que exista un error en el origen, sino más bien diferencias en las actitudes, preferencias o sentimientos. En consecuencia, es imposible llegar a un acuerdo, razón por la cual Dascal considera que es un diálogo estéril que no tiene sentido llevar a cabo.

Las controversias se ubican en un punto intermedio entre las discusiones y las disputas. Si bien se inician a raíz de un problema sobre el cual existen posiciones encontradas, en el desarrollo de estas surgen una multiplicidad de divergencias o problemas que los intervinientes pretenden resolver. Siempre hay un resultado positivo en las controversias, donde la argumentación de una de las partes se impone y entre todos deciden una forma de clausura, que no implica, necesariamente, la resolución de aspectos fundamentales o absolutos. Hay controversias que han durado decenas de años. Existen desde ya varias formas de cerrar una controversia, que difieren entre los autores.

Más allá de las críticas que pueda recibir la teoría de Dascal, tanto en lo concerniente a su metodología para el estudio de las controversias (la pragmática), como al valor que les asigna a estas en la evolución de la ciencia, no deja de ser muy interesante su idea de reconocer en ellas "el motor" del progreso en el conocimiento. A tal punto le ha dado importancia al tema, que Marcelo Dascal ha sido el fundador de una Sociedad Científica denominada "International Association for the Study of Controversies (IASC)".

Otros autores, como Ernan McMullin, han considerado que para que tenga lugar una controversia científica, partes sustanciales de la comunidad científica deben apoyar una u otra de las posiciones. De este modo toma importancia el papel de la comunidad en la determinación de una controversia, contextualizando muchas de ellas como hechos históricos que suceden en determinado lugar y fecha.

Finalmente, otro filósofo contemporáneo de origen catalán, Jordi Vallverdú, de la Universidad Autónoma de Barcelona, interesado en el tema de las controversias, define como controversia científica, toda controversia en la que participe, como mínimo, una disciplina científica de la que se cuestionen sus resulta- dos, los protocolos empleados o su aceptabilidad epistémica. Las analiza como un momento de la dinámica científica. Para ello hace una distinción entre dos niveles de esta actividad: el de la macrodinámica y el de la microdinámica científica y lo grafica en el esquema de dinámica de la ciencia que muestra la figura 1.

Según este autor, los cambios en la ciencia se producen por múltiples y pequeñas controversias que orientan las disciplinas a través del tiempo, y que se dan en un nivel de microdinámica científica. La mayor parte de los debates, polémicas o controversias científicos tienen lugar dentro de esta microdinámica científica, sin que se produzcan cambios paradigmáticos, lo que en el esquema estaría representado por cambios del estado E1 al E2, mediado por las controversias y dentro del paraguas de un paradigma. Cuando las controversias se acentúan, se genera una "crisis" que determina un cambio a mayor escala, a una modificación de la macrodinámica científica, vale decir, a un cambio de paradigma (transición de P1 a P2). Estos últimos suceden muy infrecuentemente a lo largo de la historia. Dentro de los estudios de Ciencia, Tecnología y Sociedad, ha surgido una especialidad con enfoque multidisciplinar que es el Estudio de las Controversias Científicas y Técnicas. Es en este dominio donde Vallverdú propone un modelo para el análisis de las controversias, que denomina "Campos de Controversias", con valor para el análisis concreto de controversias relativas a las ciencias, su complejidad y tipos de resolución (Fig. 2).

En él se aprecia la complejidad de factores que participan, sus múltiples interacciones, y la posibilidad de resoluciones diversas. Es evidente que resulta más fácil de resolver la controversia que se da solo en un campo, como el científico, que cuando en ella participan diversos actores o campos, siendo las más complejas las situadas en el centro con interacción de múltiples campos.

\section{Desde el punto de vista pedagógico}

La controversia es considerada actualmente como una estrategia para el desarrollo del pensamiento crítico, entendiendo como tal un conjunto de habilidades que le permiten al individuo decidir qué hacer y qué pensar sobre la base de la reflexión (Bentacourt, 2009).

El desarrollo del pensamiento crítico es un objetivo en educación, tanto para la formación de alumnos como de docentes, y la controversia, una herramienta idónea para tal fin.

Desde una perspectiva educativa, la controversia académica tiene lugar cuando las ideas, informaciones, teorías, opiniones y conclusiones de un estudiante o grupo de estudiantes difieren de las de otros y ambos tratan de llegar a un acuerdo. Vale decir, se convalida en educación el concepto positivo que tienen las controversias en el ambiente científico, como analizábamos previamente. 


\section{FIGURA 1}

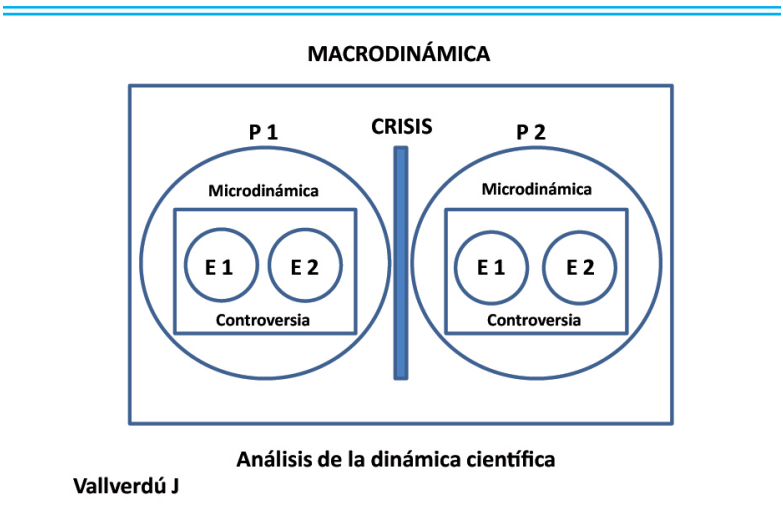

Concepción de la dinámica científica según J. Vallverdú. $\mathrm{P}=$ paradig$\mathrm{ma}, \mathrm{E}=$ estado

Es común que se promueva y aplique la enseñanza mediante controversias en numerosas disciplinas y niveles educativos. A tal efecto se han desarrollado guías y recomendaciones para su implementación.

¿Qué es lo que se pretende desarrollar mediante el ejercicio de controversias académicas en la formación de un pensamiento crítico? Hay seis habilidades básicas implicadas en este sentido: habilidad para presentar las posiciones personales; para entender las posiciones opuestas; para buscar empatía; para actuar con coherencia; para saber argumentar, y para lograr acuerdos.

El pensamiento crítico es un pensamiento disciplinado y autodirigido, que representa la perfección del pensamiento en una disciplina o dominio particular (Paul, 1990). No obstante, puede darse en dos sentidos en el ámbito de una polémica: uno débil y otro fuerte. En la versión débil, el sujeto emplea el pensamiento crítico con el oponente, pero no se somete a los mismos patrones intelectuales. Es un pensamiento que fracasa

\section{FIGURA 2}

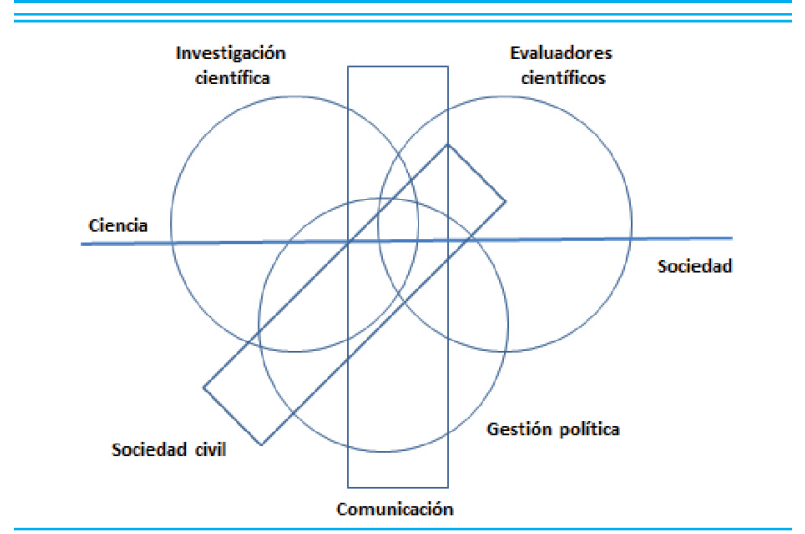

Campos de controversias (J. Vallverdú)

en la comprensión de posiciones opuestas y que pretende explotar sus habilidades con fines particulares o sectoriales excluyendo la opinión de los demás. En el sentido fuerte, aplica el pensamiento crítico a su propia argumentación, es capaz de comprender opiniones contrarias, es constructivo y aporta al bien común. Desde ya, esta es la única forma en que se puede promover el pensamiento crítico en el ámbito docente. En definitiva, el pensamiento crítico busca el desarrollo de una persona reflexiva, crítica, argumentativa, propositiva, creativa, transformadora y básicamente ética.

Pues bien, teniendo en consideración los conceptos expuestos, que de algún modo avalan el empleo de las controversias tanto desde el punto de vista filosófico como pedagógico, quiero invitarlos a aprovechar la oportunidad del Congreso para utilizar esta herramienta en la divulgación y progreso de nuestra disciplina, que es la cirugía. De este modo, doy por inaugurado el Octogésimo Octavo Congreso Argentino de Cirugía. ¡Muchas gracias! 